\title{
Efeitos de um programa de exercícios cinesioterapêuticos em idosas com osteoartrose de joelho
}

\author{
Juvêncio César Lima Assis ${ }^{1}$; Priscila Dantas Leite e Sousa² ; Elisângela Villa de Assis ${ }^{3}$; \\ Sidney Medeiros de Oliveira ${ }^{4}$; Gislene Farias de Oliveira ${ }^{5}$
}

\begin{abstract}
Resumo: O crescimento da população idosa é uma realidade presente em todo o mundo, principalmente nos países em desenvolvimento como o Brasil, doenças típicas são inúmeras destacando-se a osteoartrose (AO) também conhecida como artrose representando um dos problemas de saúde mais freqüentes em indivíduos nessa faixa etária. Trata-se de uma doença reumática, degenerativa e progressiva que acomete articulações sinoviais, promovendo alterações na cartilagem articular manifestando-se por dor, rigidez matinal de curta duração, mínimos sinais inflamatórios locais que com o tempo tornam-se intensos, crepitação, hipotrofia muscular, limitação da amplitude de movimento. Objetivo: Verificar a influência da cinesioterapia, aplicada como tratamento da AO no joelho, em pacientes idosas portadoras da doença. Métodos: Participaram do estudo 14 mulheres com idades entre 55 e 78 anos, onde todas foram submetidas a um processo de triagem e avaliação antes e após o tratamento. Foi utilizado como instrumento de coleta o questionário WOMAC específico para avaliação de qualidade de vida em indivíduos com osteoartrose de quadril e/ou joelho, sendo avaliado dor, rigidez e funcionalidade, outro instrumento de medida utilizado foi o goniômetro, avaliando a amplitude articular (ADM), sendo medidas as ADMs das flexões e extensões de ambos os joelhos. Resultados: No escore total (média) do questionário percebeu-se que os valores mantiveram-se dentro dos valores da literatura, comparando amplitudes de flexão e extensão direita e esquerda observou-se diferença significativa. Conclusão: Foi evidenciada diminuição significativa da dor, rigidez e aumento da funcionalidade em todas as participantes no presente estudo, mostrando dessa forma que realmente a cinesioterapia é eficaz no tratamento da osteoartrose.
\end{abstract}

Palavras Chave: Osteoartrose. Joelho. Fisioterapia.

\section{Effects of an exercise program cinesiotherapy in elderly with osteoarthritis of knee}

\begin{abstract}
The aging population is a present reality in the world, especially in developing countries like Brazil, typical diseases are numerous highlighting osteoarthritis (OA), also known as arthrosis representing one of the most common health problems in individuals in this age group. It is a rheumatic, degenerative and progressive that affects synovial joints, promoting changes in the articular cartilage manifesting as pain, morning stiffness of short duration, minimal local inflammatory signs that over time become intense, crackling, muscular atrophy , limitation of range of motion. Objective: To investigate the influence of kinesiotherapy applied as treatment of OA knee in elderly patients with the disease. Methods: The study included 14 women aged 55 to 78 years, where all were subjected to a screening process and evaluation before and after treatment. It was used as a tool for collecting specific WOMAC questionnaire for assessing quality of life in patients with osteoarthritis of the hip and / or knee, being evaluated pain, stiffness and function, another measurement tool used was the goniometer, assessing joint range of motion (ROM ), which measures the ADMs of crunches and stretches of
\end{abstract}

\footnotetext{
${ }^{1}$ Fisioterapeuta, graduado em Fisioterapia pela Faculdade Santa Maria (FSM), Cajazeiras,PB-Brasil, e-mail: juvêncio_178@hotmail.com ${ }^{2}$ Fisioterapeuta, graduada em Fisioterapia pela Faculdade Santa Maria (FSM), Cajazeiras,PB-Brasil, e-mail: prisciladantas.fisio@gmail.com ${ }^{3}$ Fisiotreapeuta, mestre em Ciências da Nutrição pela UFPB; Especialista em Fisioterapia Pneumofuncional pela UGF; Professora das disciplinas Fisioterapia em Terapia Intensiva; Fisioterapia Aplicada às Disfunções do Sistema Respiratório e Cardiovascular da Faculdade Santa Maria (FSM), Cajazeiras, PB-Brasil, email: elyvilar@ibest.com.br

4 Educador Físico pela Universidade federal de Pernambuco. Professor do Instituto Federal do Ceará. Doutorando em Ciências da saúde pela faculdade de Medicina do ABC - SP. Brasil. E-mail: sidneymeolli@ gmail.com;

5 Psicóloga. Doutora em Psicologia Social pela Universidade Federal da Paraíba. Professora da Faculdade de Medicina da universidade Federal do Ceará - UFC., Brasil. E-mail: gislenefarias@gmail.com.
} 
both knees. Results: Total score (mean) of the questionnaire was noticed that the values remained within the range of literature, comparing amplitudes of flexion and extension left and right there was a significant difference .conclusion: We detected significant reduction in pain, stiffness and increased functionality in all the participants in this study, thus showing that indeed kinesiotherapy is effective in the treatment of osteoarthritis.

Keywords: Osteoarthritis. Knee. Physiotherapy.

\section{Introdução}

O processo de crescimento da população idosa é uma realidade presente em todo o mundo, principalmente nos países em desenvolvimento como o Brasil, onde a população idosa representa mais de 19,6 milhões, somando cerca de 10,6\% da população total, como conseqüência há um aumento no numero de ocorrências de doenças crônicas e incapacidades relacionadas ao envelhecimento (LIMA, 2010; GRAGNOLATI, 2011).

Nesse contexto as doenças típicas são inúmeras destacando-se a osteoartrose (AO) também conhecida como artrose representando um dos problemas de saúde mais freqüentes e sintomáticos em pessoas de meia idade e idosos. (SEDA e SEDA, 2011; VASCONCELOS, 2006; FURTADO, ARAÚJO, ALVES e FERNANDES, 2009). Trata-se de uma doença reumática, degenerativa e progressiva que acomete articulações sinoviais, promovendo alterações na cartilagem articular manifestando-se por dor, rigidez matinal de curta duração, mínimos sinais inflamatórios locais que com o tempo tornam-se intensos, crepitação, hipotrofia muscular, limitação da amplitude de movimento (QUEIROZ, 2006; CARVALHO, 2006). Os mecanismos etiopatogênicos afetam preferencialmente a cartilagem articular formada por colágeno, proteoglicanos e glicoproteínas sintetizadas pelos condrócitos, que por sua vez desempenham uma dupla função: síntese e degradação da matriz cartilaginosa através da produção de inibidores da metaloprotease e plasminogênio. Portanto há, normalmente, um equilíbrio entre a síntese e a degradação da cartilagem, mantendo-se a homeostase tecidual. Porém, caso ocorra o desequilíbrio, dá-se início ao processo artrósico QUEIROZ, 2006; LAGO, 2003).

Os fatores de risco da osteoartrose podem ser classificados em sistêmicos e locais pela importância na ativação da ação catabólica na cartilagem (GARSTANG e STITIK, 2006). Como fatores de risco sistêmico temos a idade, etnia, sexo e características hormonais, genética, densidade óssea e fatores nutricionais. Relativamente aos fatores locais consideram-se as lesões articulares, obesidade, ocupação, esportes, biomecânica da articulação e força muscular ( SILVA, MONTANDON e CABRAL, 2008).

Id en line Revista de Psicologia. Ano 7, No. 21, Novembroo/2013 - ISSN 1981-1179. Edição eletrônica em http://idonline.emnuvens.com.br/id 
A prevalência aumenta com a idade avançada, afetando mais de $75 \%$ das pessoas acima de 65 anos de idade, e $10 \%$ da população com mais de 60 anos possuem limitação física por OA. Estima-se que $4 \%$ da população brasileira possuam OA, sendo o joelho uma das articulação mais acometida pela doença, com $37 \%$ dos casos. Afeta ambos os sexos, embora exista maior incidência em mulheres (acima dos 50 anos de idade) nas mãos, joelhos e pés influenciados pelos fatores hormonais e alterações morfológicas presentes nesta população (SEDA e SEDA, 2011; VASCONCELOS, 2006; FURTADO, ARAÚJO, ALVES e FERNANDES, 2009). Nessa direção, dentre os tratamentos que são realizados na osteoartrose, vê-se que a fisioterapia dispõe de vários recursos que podem ser utilizados como ferramenta de intervenção já que possuem finalidades positivas no seu tratamento, frente ao quadro clínico da patologia, promovendo analgesia, incremento na circulação e aumento da amplitude articular, prevenção da perda do trofismo, e força muscular através dos agentes físicos e químicos destacando a Cinesioterapia e a Eletrotermoterapia, permitindo assim, além do alívio da dor e melhor capacidade funcional, uma melhor qualidade de vida e integração no meio social.

Diante do exposto, o objetivo desse trabalho foi investigar os efeitos de um programa de exercícios cinesioterapêuticos em idosas com osteoartrose de joelho.

\section{Método}

Trata-se de um ensaio clínico não controlado com delineamento pré teste e pós teste, desenvolvido no Centro de Reabilitação de Fisioterapia pertencente à Policlínica Orcino Guedes do município de Cajazeiras - PB.

Participaram do estudo 14 mulheres com idades entre 55 e 78 anos, onde todas foram submetidas a um processo de triagem e avaliação antes e após o tratamento. Adotou-se como critérios de inclusão: Sexo feminino (já que essa população possui maior incidência de OA), paciente com diagnóstico clínico de osteoartrose de joelho segundo os critérios do American College of Rheumatology: dor, osteófitos visíveis ao raio-x e pelo menos um dos seguintes critérios: idade superior a 50 anos, rigidez matinal ou crepitação (American College of Rheumatology, 2000), onde optou-se nesse estudo pelo critério em relação à idade. Os critérios de exclusão utilizados foram: Problemas cardiovasculares, cognitivos ou outro tipo de doença reumática (artrite reumatóide), prática de exercícios físicos regulares ou tratamento da patologia há pelo menos 6 meses, cirurgia prévia do joelho, locomoção exclusiva por cadeiras de roda, uso de prótese de joelho e/ou quadril.

As participantes foram devidamente orientadas e esclarecidas acerca dos objetivos e métodos do estudo, e posteriormente assinaram o Termo de Consentimento Livre e Esclarecido (TCLE).

Id en lime Revista de Psicologia. Ano 7, No. 21, Novembroo/2013 - ISSN 1981-1179.

Edição eletrônica em http://idonline.emnuvens.com.br/id 
Foi utilizado como instrumento de coleta o questionário WOMAC específico para avaliação de qualidade de vida em indivíduos com osteoartrose de quadril e/ou joelho. Utilizou-se a versão adaptada para a população brasileira que dispõe de 24 perguntas divididas em três domínios: DOR (5 questões com faixa de pontuação de 0-20), RIGIDEZ (2 questões, com faixa de pontuação 0-8) e FUNCIONALIDADE (17 questões, com faixa de pontuação 0-68). Para cada uma das respostas há um escore especifico (nenhuma - 0 , pouca -1 , moderada -2 , intensa -3 , muito intensa -4 pontos). Para se chegar a um escore final, somam-se os valores de todas as voluntárias em cada questão, obtém-se a média, e os valores são apresentados para cada domínio(IVANOVICH, 2002; GOMES, 2007; FERNANDES, 2003).

Outro instrumento de medida utilizado foi o goniômetro, avaliando a amplitude articular (ADM), sendo medidas as ADMs das flexões e extensões de ambos os joelhos no início e no fim do tratamento. Para a realização da medição, as voluntárias foram posicionadas em decúbito ventral. O fulcro foi alinhado no côndilo femoral externo, o braço fixo paralelo a linha média externa da coxa, passando pelo trocanter e côndilo externo e o braço móvel paralelo a linha média externa da perna, em direção ao maléolo externo. O valor da Goniometria de cada paciente foi comparado ao valor de normalidade de $0-140^{\circ}$ (FERNANDES, 2003).

O mesmo programa de tratamento foi realizado em todas as voluntárias participantes, composto de: Exercícios Isotônicos (demanda movimento articular, sendo mais efetivo na aquisição de massa muscular e melhora da resistência) que foram precedidos por alongamentos dos membros inferiores (ísquiostibiais, quadríceps, tríceps sural, adutores e abdutores) durante três séries de 30 segundos. Além disso, realizou-se também mobilizações patelares e bombeamento tíbio-társico com o número de repetições, frequência e resistência pré-determinados. Os atendimentos aconteciam 2 vezes por semana durante 5 semanas totalizando 10 sessões de 50 minutos cada iniciadas em outubro de 2012.

A análise dos dados se deu através da estatística descritiva simples (média e desvio padrão) de cada uma das variáveis em estudo: score parcial da dor, rigidez e limitação funcional e score total do WOMAC e amplitude articular. Como forma de analisar a eficácia do protocolo de tratamento entre as voluntárias do estudo utilizou-se o teste t-student permitindo inferir sobre a igualdade de médias das amostras que foram analisadas antes e depois da intervenção. Para todos os procedimentos estatísticos, o nível de significância admitido foi $\mathrm{p} \leq 0,05$, utilizando o programa estatístico SPSS (Statistical Package for the Social Science), versão 16.0. 


\section{Resultados}

TABELA 1 - Avaliação da qualidade de vida das voluntárias pré e pós tratamento fisioterapêutico.

\begin{tabular}{ccccc}
\hline $\begin{array}{c}\text { QUESTIONÁRIO } \\
\text { WOMAC }\end{array}$ & $\begin{array}{c}\text { MÉDIA E DESVIO } \\
\text { PADRÃO PRÉ- } \\
\text { TRATAMENTO } \\
(\mathbf{n = 1 4 )}\end{array}$ & $\begin{array}{c}\text { MÉDIA E DESVIO } \\
\text { PADRÃO PÓS- } \\
\text { TRATAMENTO } \\
(\mathbf{n}=\mathbf{1 4})\end{array}$ & $\begin{array}{c}\text { DIFERENÇA } \\
\text { ENTRE AS } \\
\text { MÉDIAS }\end{array}$ & P \\
\hline DOR & $11,71 \pm 1,26$ & $9,14 \pm 1,5$ & 2,57 & 0,001 \\
RIGIDEZ & $7,07 \pm 0,82$ & $5,57 \pm 0,85$ & 1,5 & 0,001 \\
FUNCIONALIDADE & $23,79 \pm 3,14$ & $20,36 \pm 3,27$ & 3,43 & 0,001 \\
\hline
\end{tabular}

A Tabela 1 representa a avaliação da qualidade de vida das voluntárias em estudo. Realizou-se uma comparação de todos os domínios (dor, rigidez, funcionalidade) antes do início do programa de exercícios (pré tratamento) e após a realização de 10 sessões de exercícios (pós tratamento). A diferença entre as médias de cada domínio do questionário WOMAC apresentou diferença estatisticamente significativa $(\mathrm{p}=0,001)$.

TABELA 2 - Amplitude de movimento do joelho no pré e pós tratamento fisioterapêutico.

\begin{tabular}{clccc}
\hline \multirow{2}{*}{ GONIOMETRIA } & & $\begin{array}{c}\text { PRÉ- } \\
\text { TRATAMENTO } \\
(\mathbf{n}=\mathbf{1 4})\end{array}$ & $\begin{array}{c}\text { PÓS- } \\
\text { TRATAMENTO } \\
(\mathbf{n}=\mathbf{1 4})\end{array}$ & P \\
\hline \multirow{2}{*}{ FLEX̃̃O } & DIREITA & $126 \pm 5,9$ & $132 \pm 3,75$ & 0,001 \\
& ESQUERDA & $127 \pm 9,9$ & $132 \pm 6,68$ & 0,001 \\
\multirow{2}{*}{ EXTENSÃO } & & & & \\
& DIREITA & $1,8 \pm 2,0$ & $0,71 \pm 1,2$ & 0,005 \\
& ESQUERDA & $2,2 \pm 2,2$ & $1 \pm 1,17$ & 0,003 \\
\hline
\end{tabular}

Na comparação das amplitudes de flexão e extensão direita e esquerda observou-se diferença significativa com $\mathrm{p}=0,001$ para flexão dos dois joelhos, e $\mathrm{p}=0,005$ para extensão do joelho direito e $p=0,003$ para extensão do joelho esquerdo.

\section{Discussão}

Os resultados do presente estudo mostraram que houve diminuição da dor, rigidez e aumento da funcionalidade em todas as voluntárias quando comparados os valores antes e após o tratamento. Foi evidenciado também que houve aumento da amplitude de movimento de flexão do membro direito e esquerdo quando comparado o antes e depois da intervenção. 
http://idonline.emnuvens.com.br/id ISSN on-line: 1981-1179

Tais resultados corroboram com as investigações realizadas por Lago (2005) que, utilizando o questionário WOMAC em idosos que apresentam gonartrose, evidenciou no seu estudo, redução da dor e da rigidez após realização de exercícios específicos e direcionados. No mesmo sentido, Estudos de Limeira (2009) evidenciou que a funcionalidade está inteiramente relacionada com a redução da dor, e posteriormente ao ganho da amplitude de movimento, e que a cinesioterapia é um fator indispensável no que se refere ao ganho de função e retorno as atividades da vida diária (Limeira, 2009).

Amplitude articular das idosas avaliadas no seu estudo aumentou após a realização da cinesioterapia (ALMEIRA, 2012) corroborando dessa forma com nosso estudo. A limitação do movimento articular pode acentuar a degeneração da cartilagem articular, dado esta necessitar de uma amplitude articular normal para garantir a sua integridade e nutrição através do líquido sinovial ao longo de toda a sua superfície, ou seja, a amplitude de movimento da articulação do joelho deve ser preservada ao máximo para se garantir um bom funcionamento, portanto, a realização de exercícios direcionados é de suma importância (ESPANHA, PASCOAL, CORREIA e SILVA, 2004). Importante ressaltar também, que a diminuição da força é um dos sintomas que mais comprometem a função dos idosos com osteoartrose, afetando diretamente na realização das atividades diárias, e muitas vezes limitando a capacidade de desempenhar as mesmas, acelerando de forma decisiva o desenvolvimento da doença (LAGO, 2005). No mesmo sentido, há uma redução na força do aparelho extensor do membro inferior dos participantes, evidenciando uma diminuição da capacidade para realizar a marcha, devido à fadiga muscular, aumentando o stress articular, promovendo o desenvolvimento da gonartrose e comprometendo a função "amortecedora" destes músculos; esta por sua vez desperta a dor, reduzindo a capacidade para realizar a marcha (EVEIK e SONEL, 2002).

Estudo realizado com idosas evidenciou uma melhora significativa da dor e rigidez articular após a realização de um programa de tratamento que consistiu na combinação de exercícios isotônicos e alongamentos, sendo constatado que todas as participantes obtiveram melhora da dor e diminuição da rigidez articular (ALMEIDA, 2012).

Os resultados obtidos confirmam o que é defendido por Bellamy (2003) e Grabiner (2004), segundo o qual a dor tende a limitar ou diminuir os movimentos articulares, o que também vai ter implicações ao nível da capacidade funcional do idoso com gonartrose, sendo de fundamental importância a realização de exercícios específicos permitindo assim um aumento da amplitude articular, prevenção da perda do trofismo, e ganho de força muscular.

Id en line Revista de Psicologia. Ano 7, No. 21, Novembroo/2013 - ISSN 1981-1179. Edição eletrônica em http://idonline.emnuvens.com.br/id 


\section{Conclusão}

Foi evidenciada diminuição significativa da dor, rigidez e aumento da funcionalidade em todas as participantes no presente estudo, mostrando dessa forma que realmente a cinesioterapia é eficaz no tratamento da osteoartrose. Mesmo com as diferenças verificadas comparativamente com outros estudos, é difícil generalizar o escore total de WOMAC, uma vez que, este depende de três domínios específicos dor, rigidez e limitação funcional. Apesar de esse estudo contribuir para o embasamento clínico de fisioterapeutas e demais profissionais da área de saúde, o mesmo, apresenta limitações, dado o reduzido número de casos, e a não verificação dos efeitos a longo prazo, sendo assim, são necessários mais estudos na área.

\section{Referências}

ALMEIDA JF. Efeito de dois tratamentos fisioterapêuticos em mulheres idosas com osteoartrose do joelho [dissertação]. Maranhão: Universidade federal do maranhão-UFM; 2012.

BELLAMY N. Womac Osteoarthritis Index. User Guide VI. WOMAC®; 2003.

CARVALHO ÉR. Tratamento clínico fisioterapêutico em pacientes idosos com gonoartrose. Anais I simpósio do instituto de geriatria e gerontologia JEM e VI Jornada gerontológica da Pontifícia Universidade Católica de São Paulo; 5-9 de abril 2006; São Paulo-SP, Brasil; 2006.

ESPANHA M. PASCOAL A. CORREIA P. SILVA P. Noções Fundamentais de fisiologia articular: Anatomofisiologia, Sistema osteoarticular. 2 ed. Portugual: Lisboa; 2004. p. 51-80.

EVCIK D. Sonel B. Effectiveness of a Home-based Exercise Therapyand Walking Program On Osteoarthritis of the Knee. 1 ed. v. 2, p.30-41, 2002.

FERNANDES MI. Tradução e validação do questionário de qualidade de vida específico para osteoartrite - WOMAC (Western Ontário and Mcmaster Universities) para língua portuguesa. Unifesp, Escola Paulista de Medicina, Universidade Federal de São Paulo. São Paulo, Brasil; 2003. p. 1- 100 . 
FURTADO FJA. ARAÚJO ERA. ALVES LCM; FERNANDES JF. Efeitos de dois tratamentos fisioterapêuticos em pacientes idosos com gonartrose: avaliação através dos níveis de hidroxiprolina na excreção da urina Rib - Revista de Investigação Biomédica do Uniceuma. v.1. p.83-96, 2009.

GARSTANG SV. STITIK TP. Osteoarthritis: epidemiology, risk factors, and pathophysiology. Am J Phys Med Rehabil. v.85(11 Suppl):S2-11, 2006.

GOMES WF. Impacto de um programa estruturado de fisioterapia aquática em idosas com osteoartrite de joelho [dissertação]. Belo Horizonte: Minas Gerais: Universidade Federal de Minas Gerais; 2007.

GRABINER MD. (2004). Obesity and Lower Extremity Osteoarthritis: Is Body Mass Destiny. National Association for Physical Education Higher Education. p. 56,41-49, 2004.

GRAGNOLATI M. Envelhecendo em um Brasil mais velho. [periodico na internet] 2011 [citado em julho 2011]. Disponível em: http://siteresources.worldbank.org/BRAZILINPOREXTN/Resources/38171661302102548192/Envelh ecendo_Brasil_Sumario_Executivo.pdf.

IVANOVITH MF. Tradução e validação do questionário de qualidade de vida específico para osteoartrose WOMAC (Wester Ontario and McMaster Universities) para a língua portuguesa [dissertação]. São Paulo: Universidade Federal de São Paulo; 2002.

LAGO RL. Efeitos de um Programa de Exercício em Idosos Institucionalizados com Gonartrose [dissertação]. Portugual: Universidade do Porto e Faculdade de Ciências do Desporto e de Educação Física; 2005.

LIMA T V. Humanização na Atenção à Saúde do Idoso. Rev Sociedade Saúde. v.19. n.4. p.866-877, 2010.

LIMEIRA SC. Benefícios dos exercícios contra-resistidos para idosos com osteoartrose do joelho [monografia]. Brasília: Universidade Gama Filho: Pós graduação em educação física; 2009. 
http://idonline.emnuvens.com.br/id

ISSN on-line: 1981-1179

QUEIROZ LF. Efeitos da Hidroterapia em Pacientes Idosos com Osteoartrose de Joelhos. Rev Terapia Manual. v.16. n.4. p.50-52, 2006.

SEDA H. SEDA AC. Osteoartrite. In: Moreira C, Carvalho MAP. Reumatologia: diagnóstico e tratamento. Rio de Janeiro; 2 ed; Editora Médica e Científica. p. 289-307, 2001.

SILVA NA. Montandon ACOS. Cabral MVSP. Doenças osteoarticulares degenerativas periféricas. v.6 (Supl 1): S21-S8, 2008.

VASCONCELOS KSS. Relação entre intensidade de dor e capacidade funcional em indivíduos obesos com osteoartrose de joelho. Rev. Bras. Fisioterapia. v.10. n.2. p. 213-218, 2006.

\section{Como citar este artigo (Formato ISO):}

ASSIS, J.C.L.; SOUSA, P.D.L.; ASSIS, E.V.; OLIVEIRA, S.M.; OLIVEIRA, G.F. Efeitos de um programa de exercícios cinesioterapêuticos em idosas com osteoartrose de joelho. Id on Line Revista de Psicologia, Novembro de 2013, vol.1, n.21, p. 45-53. ISSN 1981-1189. 